\title{
Structure and dynamics of nanoemulsions: Insights from combining dynamic and static neutron scattering
}

\author{
I. Hoffmann* and P. Heunemann ${ }^{\dagger}$ \\ Institut Max von Laue-Paul Langevin (ILL), F-38042 Grenoble Cedex 9, France and Stranski-Laboratorium für Physikalische und \\ Theoretische Chemie, Institut für Chemie, Technische Universität Berlin, Straße des 17. Juni 124, Sekr. TC 7, D-10623 Berlin, Germany \\ B. Farago and I. Grillo \\ Institut Max von Laue-Paul Langevin (ILL), F-38042 Grenoble Cedex 9, France
}

O. Holderer

Jülich Center for Neutron Science, FRM II Garching, Lichtenbergstr. 1, D-85747 Garching, Germany

M. Päch

Fraunhofer-Institut für Angewandte Polymerforschung IAP,Geiselbergstr. 69, D-14476 Potsdam, Germany

\author{
M. Gradzielski $i^{\ddagger}$ \\ Stranski-Laboratorium für Physikalische und Theoretische Chemie, Institut für Chemie, Technische Universität Berlin, \\ Straße des 17. Juni 124, Sekr. TC 7, D-10623 Berlin, Germany
}

(Received 3 October 2012; published 17 December 2012)

\begin{abstract}
Despite their lack of thermodynamical stability, nanoemulsions can show a remarkable degree of kinetic stability. Among the various different preparation methods the phase-inversion concentration method is particularly interesting as it occurs spontaneously. Here we investigate such a system composed of a surfactant, cosurfactant, and oil that upon dilution with water forms long time metastable oil-in-water nanoemulsion droplets. The dynamics of the amphiphilic monolayers and its elastic properties is important for their stability and therefore the monolayer dynamics have been investigated by neutron spin echo (NSE). Despite the difficulties arising from the inherently polydisperse nature and the large number of different components necessarily contained in commercial nanoemulsion formulations, information concerning the membrane rigidity was extracted from the combination of small angle neutron scattering and NSE and several different formulations are compared. These results show that small amounts of different admixed ionic surfactants can modify the monolayer rigidity substantially and similarly effects of surface bound polyelectrolytes have been evaluated.
\end{abstract}

DOI: 10.1103/PhysRevE.86.061407

PACS number(s): $82.70 . \mathrm{Kj}, 25.40 . \mathrm{Dn}$

\section{INTRODUCTION}

Nanoemulsions differ from microemulsions by the fact that in contrast to the latter they are not thermodynamically stable. Nonetheless nanoemulsion droplets, being typically in the size range of $20-100 \mathrm{~nm}$, are only somewhat larger than microemulsion droplets and a thorough structural characterization of nanoemulsions is a central aspect for the understanding of their stability, where so far the criteria for having kinetically stabilized nanoemulsions are still not really established. Nanoemulsions are conventionally formed either by higher-energy input methods (high-pressure homogenization, ultrasonication, etc.) $[1,2]$ but low-energy methods such as the phase inversion temperature (PIT) method [3-5] or the phase inversion concentration (composition) (PIC) method [6-9] have been employed increasingly in recent times. Especially the PIC method, where one obtains a nanoemulsion

\footnotetext{
*hoffmann@ill.fr

${ }^{\dagger}$ Present address: Inst. f. Lebensmittel- u. Ernährungs wissenschaften LFO E21 Schmelzbergstrasse 98092 Zürich, Switzerland.

${ }^{\ddagger}$ michael.gradzielski@tu-berlin.de
}

simply by diluting an appropriate surfactant-oil mixture with water is very attractive as it is a very mild method for obtaining nanoemulsions in a very simple fashion. However, so far the requirements on the molecular composition of the surfactant-oil mixture are far from being clear and as well the mechanism of formation and even the structural details about the nanoemulsion droplets are not really clear. The other key issue apart from the formation process is the stability of the formed nanoemulsions as they are prone to Ostwald ripening and coalescence processes. For the classical case of nanoemulsion or miniemulsion formulation an osmotic agent is added, which is insoluble in the continuous phase [for instance squalene or polyethylene in the case of oil-inwater (OW) nanoemulsions] and thereby Ostwald ripening is effectively suppressed due to the osmotic pressure that is building up in the droplets that become smaller in the classical Ostwald ripening process [10-12]. The other mechanism of emulsion ripening, which cannot be prevented by an osmotic agent, is coalescence. Here, as well as for the dynamics in microemulsions, it can be assumed that the bending rigidity of the surfactant film plays a key role in determining the rate of coalescence $[13,14]$. Accordingly the bending modulus of the surfactant monolayer of a nanoemulsion is an important quantity for understanding its stability. 
In a recent investigation [9] we studied the formation of nanoemulsions based on polyglyceryl-4 laurate/dilauryl citrate as surfactant and bis(2-ethylhexyl) carbonate-phenoxyethanolparabens as oil, which are long-time stable even without the addition of an osmotic agent. Below a concentration of surfactant-oil of $20 \mathrm{wt} \%$ metastable nanoemulsion droplets are formed with an average droplet radius of 20-40 nm. Structural investigations by means of cryotransmission electron microscopy (cryo-TEM), small-angle neutron scattering (SANS), and dynamic light scattering (DLS) interestingly pointed to the presence of a bimodal size distribution containing rather small droplets of 12-15 $\mathrm{nm}$ radius and larger ones of 30-40 nm radius, which are present at the same time. To gain further insight into the system and to improve our understanding of the mechanisms governing the stability of nanoemulsions, combined SANS and NSE measurements have been performed on several nanoemulsion formulations, investigating if and how an increased concentration of charged surfactant and the addition of oppositely charged polyelectrolyte influences the rigidity of the nanoemulsion membrane, as that for sure is of importance for controlling the coalescence in such systems. An additional advantage of neutron scattering in that respect is the possibility to employ contrast variation and this is particularly useful for nanoemulsions in the shell contrast, where the information about the dynamics of the droplets is much more pronounced than in the case of bulk contrast [15]. By this combination of static information with complementary dynamic information on these nanoemulsions a much more refined picture can be deduced and it demonstrates the power of neutron scattering to deduce information regarding the properties of the important class of nanoemulsion, which is not similarly accessible by other methods.

\section{THEORETICAL BACKGROUND}

\section{A. Small-angle neutron scattering (SANS)}

The scattering intensity $I(Q)$ of polydisperse droplets observed in SANS can in general be described as

$$
I(Q)={ }^{1} N S(Q) \int_{0}^{\infty} f\left(R, x_{1,2, \ldots, i}\right) P\left(Q, R, \Delta S_{\mathrm{LD}}\right) d R+I_{\mathrm{bkg}},
$$

where ${ }^{1} N$ is the number density of aggregates, $\Delta S_{\mathrm{LD}}$ the difference of scattering length density (SLD) between the droplets and the average SLD of the solution, $S(Q)$ the effective structure factor accounting for interparticle interferences, $f\left(R, x_{1,2, \ldots, i}\right)$ the normalized size distribution function, which depends on radius $R$ and parameters $x_{1,2, \ldots, i}$, $I_{\mathrm{bkg}}$ the incoherent background, $P(Q, R)$ the form factor, and $Q$ is the vector of momentum transfer $Q=4 \pi / \lambda \sin (\theta / 2)$, with wavelength $\lambda$ and scattering angle $\theta$. The scattering amplitude of a sphere is given by

$$
F\left(Q, R, \Delta S_{\mathrm{LD}}\right)=\frac{4 \pi R^{3}}{3} \Delta S_{\mathrm{LD}} 3 \frac{\sin (Q R)-Q R \cos (Q R)}{(Q R)^{3}},
$$

and the form factor of a homogeneous sphere is $P_{s}\left(Q, R, \Delta S_{\mathrm{LD}}\right)=F\left(Q, R, \Delta S_{\mathrm{LD}}\right)^{2}$, whereas for a sphere with core-shell structure it reads

$$
\begin{aligned}
& P_{\mathrm{sh}}\left(Q, R, R_{s}, \Delta S_{\mathrm{LD}, 1}, \Delta S_{\mathrm{LD}, 2}\right) \\
& \quad=\left[F\left(Q, R, \Delta S_{\mathrm{LD}, 1}\right)-F\left(Q, R-R_{s}, \Delta S_{\mathrm{LD}, 2}\right)\right]^{2},
\end{aligned}
$$

where $R$ is the overall radius of the sphere, $R_{s}$ is the thickness of the shell, $\Delta S_{\mathrm{LD}, 1}$ is the difference in scattering length density between the shell and the solvent and $\Delta S_{\mathrm{LD}, 2}$ is the difference between shell and core.

The normalized size distribution function $f\left(R, x_{1,2, \ldots, i}\right)$ can be of any type but for our purposes we focused on a bimodal log-normal distributions, which has previously been shown to fit data from such systems [9]. The normalized log-normal distribution function is defined as

$$
f(x, \mu, \sigma)=\frac{1}{\sqrt{2 \pi} \sigma x} \exp \left(-\frac{[\ln (x)-\mu]^{2}}{2 \sigma^{2}}\right),
$$

where the parameters $\mu$ and $\sigma$ are the mean value and the standard deviation of the logarithm of $x$. The arithmetic mean value of the distribution is $M=\exp \left(\mu+1 / 2 \sigma^{2}\right)$, the standard deviation is $S_{D}=\sqrt{\left[\exp \left(\sigma^{2}\right)-1\right]} \exp \left(\mu+1 / 2 \sigma^{2}\right)$. The particle number density can be rewritten as a function of the volume fraction and the size distribution, for spheres

$$
{ }^{1} N(\phi, \mu, \sigma)=\frac{\phi}{\int_{0}^{\infty} 4 \pi / 3 R^{3} f(R, \mu, \sigma) d R} .
$$

Thus, for a bimodal log-normal distribution of spheres with $S(Q)=1$ and assuming that both modes have the same SLD

$$
\begin{aligned}
I(Q)= & { }^{1} N\left(\phi_{1}, \mu_{1}, \sigma_{1}\right) \int_{0}^{\infty} f\left(R, \mu_{1}, \sigma_{1}\right) P_{s}\left(Q, R, \Delta S_{\mathrm{LD}}\right) d R \\
& +{ }^{1} N\left(\phi_{2}, \mu_{2}, \sigma_{2}\right) \int_{0}^{\infty} f\left(R, \mu_{2}, \sigma_{2}\right) \\
& \times P_{s}\left(Q, R, \Delta S_{\mathrm{LD}}\right) d R+I_{\mathrm{bkg}},
\end{aligned}
$$

where $\phi=\phi_{1}+\phi_{2}$ is the volume fraction of spheres. An analogous expression [with $P_{\mathrm{sh}}\left(Q, R, R_{s}, \Delta S_{\mathrm{LD}, 1}, \Delta S_{\mathrm{LD}, 2}\right)$ ] holds for spheres with a core-shell structure. Since a significant part of the material (in particular the cosurfactant) is in the bulk solution a factor $f$ has to be introduced such that the volume fraction of droplets is related to the macroscopic volume fraction $\phi_{m}$ by $\phi=\phi_{m}(1-f)$. Furthermore, the fraction of $\phi$ that is in $\phi_{1}$ is expressed in terms of $\phi_{1 \text { rel }}$, such that $\phi_{1}=\phi \phi_{1 \text { rel }}$ and $\phi_{2}=\phi\left(1-\phi_{1 \mathrm{rel}}\right)$.

\section{B. Neutron spin echo (NSE)}

Neutron spin echo allows the direct access of the intermediate scattering function $s(Q, t)$. The normalized intermediate scattering function due to diffusion of a single species is

$$
S(Q, t)=s(Q, t) / s(Q, 0)=\exp \left(-D Q^{2} t\right) .
$$

According to the Stokes-Einstein equation, the diffusion coefficient $D$ of a sphere is related to its radius $R$ by $D=\frac{k_{B} T}{6 \pi \eta R}$, with Boltzmann's constant $k_{B}$, temperature $T$, and solvent viscosity $\eta$. If the sample is polydisperse or dynamics other than simple translational diffusion are observed, often $S(Q, t)$ can still be described in a similar manner with a $Q$-dependent effective diffusion coefficient $D_{\text {eff }}(Q)$. 
For a bimodal distribution of diffusing spheres, the exact expression for the intermediate scattering function reads

$$
\begin{aligned}
S(Q, t)= & \frac{{ }^{1} N\left(\phi_{1}, \mu_{1}, \sigma_{1}\right)}{I(Q)} \int_{0}^{\infty} f\left(R, \mu_{1}, \sigma_{1}\right) \\
& \times P_{s}\left(Q, R, \Delta S_{\mathrm{LD}}\right) \exp \left[-D(R) Q^{2} t\right] d R \\
& +\frac{{ }^{1} N\left(\phi_{2}, \mu_{2}, \sigma_{2}\right)}{I(Q)} \int_{0}^{\infty} f\left(R, \mu_{2}, \sigma_{2}\right) \\
& \times P_{s}\left(Q, R, \Delta S_{\mathrm{LD}}\right) \exp \left[-D(R) Q^{2} t\right] d R .
\end{aligned}
$$

An additional component to the decay of $S(Q, t)$ comes from the dynamics of the droplet membrane. This can be described in terms of the theory developed by Zilman and Granek for the undulations of flat membranes [16,17]. Starting from a Helfrich bending Hamiltonian [18]

$$
H=\frac{1}{2} \kappa \int\left[\nabla_{r}^{2} h(\vec{r})\right]^{2} d r,
$$

with the the undulation amplitude $h(\vec{r})$, they predict a stretched exponential behavior for the intermediate scattering function with a stretch exponent of $2 / 3$

$$
S(Q, t)=\exp \left[-\left(\Gamma_{K} Q^{3} t\right)^{2 / 3}\right]
$$

with $\Gamma_{K}=0.025 \gamma \sqrt{\frac{k T}{\kappa}} \frac{k T}{\eta}$, with the bending modulus of the membrane $\kappa$ and the viscosity of the solvent $\eta$. To first order $\gamma \approx 1-3 k_{B} T /(4 \pi \kappa) \ln (Q \xi)$, so $\gamma \approx 1$ for sufficiently large $\kappa . \xi$ is a length on the order of the size of the objects (i.e., the droplets in our case). The absolute values obtained for $\kappa$ with NSE applying the above formula are known to be wrong in many cases [19,20] and some attempts have been made to correct the problem [19,21]. For example, the approach of Richter et al. [19] explicitly takes into account the dependence on the length scale considered. The resulting formula makes it necessary to evaluate some nested integrals and the value of $\kappa$ as a function of length scale and relaxation frequency has been mapped in a range of parameters. Watson and Brown [21] adapted the theory of Seifert and Langer [22], which involves contributions from lateral flow in bilayers and interbilayer friction in addition to the bending energy of the membrane to the framework of the Zilman-Granek model. As a somewhat more straightforward approach using an effective viscosity $\eta_{\text {eff }}=3 \eta$ instead of the macroscopic solvent viscosity to account for dissipation between the solvent and the membrane has proven to yield good results [20,23]. For monolayers surrounded by two different solvents (e.g., oil and water as in our case or in microemulsion sponge phases) the average of the two viscosities may be used instead of the solvent viscosity [24] to obtain more realistic values of $\kappa$. In any case, the dissipation of energy via the viscosity of the solvent(s) is an issue that will cause some uncertainty and even more so the dissipation of energy within the membrane/monolayer that typically is not considered at all. In general, some caution should be taken with regard to absolute values of $\kappa$. In contrast, the relative changes deduced can be considered to be reliable.

So, if in addition to simple diffusion undulations of the membrane of the larger droplets $\left(\mu_{1}<\mu_{2}\right)$ are observed,
$S(Q, t)$ becomes

$$
\begin{aligned}
S(Q, t)= & \frac{{ }^{1} N\left(\phi_{1}, \mu_{1}, \sigma_{1}\right)}{I(Q)} \int_{0}^{\infty} f\left(R, \mu_{1}, \sigma_{1}\right) P_{s}\left(Q, R, \Delta S_{\mathrm{LD}}\right) \\
& \times \exp \left[-D(R) Q^{2} t\right] d R+\frac{{ }^{1} N\left(\phi_{2}, \mu_{2}, \sigma_{2}\right)}{I(Q)} \\
& \times \int_{0}^{\infty} f\left(R, \mu_{2}, \sigma_{2}\right) P_{s}\left(Q, R, \Delta S_{\mathrm{LD}}\right) \\
& \times \exp \left[-D(R) Q^{2} t\right]
\end{aligned}
$$

where the parameter $a(Q)$ describes the ratio between the signal coming from membrane dynamics and the signal coming from simple diffusion for the larger-sized distribution. Even though this may look like a somewhat complicated expression at first, it should be kept in mind that the only free parameters here are in fact $a(Q)$ and $\Gamma_{k}$, since the size distribution is known from SANS. Also, the use of the ZilmanGranek model, which has been developed for flat membranes, may seem to lack justification, but since the droplets we are dealing with here are quite large the surface appears as almost flat in the relevant $Q$ range. For instance, a $Q$ value of $11 / \mathrm{nm}$ corresponds to a length scale of $l=2 \pi / Q \approx 6 \mathrm{~nm}$, which in turn corresponds to only about $6 \mathrm{~nm} /(18 \mathrm{~nm} \cdot 2 \pi) \approx 5 \%$ of the circumference of a circle with a radius of $18 \mathrm{~nm}$.

\section{MATERIALS AND METHODS}

\section{A. Materials}

The oil-surfactant mixture Tego Wipe $\mathrm{DE}^{\circledR}$ consists of $66 \mathrm{wt} \%$ di(2-ethylhexyl) carbonate [DEHC, $\left(\right.$ Tegosoft ${ }^{\circledR}$ Evonic Industries)], 12 wt\% of Euxyl ${ }^{\circledR}$ K300 (Schülke and Mayr $\mathrm{GmbH}$ ) and $21.12 \mathrm{wt} \%$ polyglycerol-4 laurate and 0.88 wt \% dilauryl citrate (Tego ${ }^{\circledR}$ Care PL 4, Evonic Industries, see Fig. S1 and Fig. S2 [25]), where Tegosoft ${ }^{\circledR}$ and Euxyl ${ }^{\circledR}$ $\mathrm{K} 300$ are the oil components (where the phenoxyethanol and the parabens can be considered like a cosurfactant in that system) and $\mathrm{Tego}^{\circledR}$ Care PL 4 is the surfactant component.

Euxyl $^{\circledR}$ K300 consists of 72 wt\% phenoxyethanol and several different parabens [methyl- (16 wt $\%)$, ethyl- (4 wt $\%)$, propyl- $(2 \mathrm{wt} \%)$, butyl- $(4 \mathrm{wt} \%)$ and isobutylparabene (2 wt\%)]. The compositions of Euxyl ${ }^{\circledR}$ K300 and Tego Wipe $\mathrm{DE}^{\circledR}$ are summarized in tables $\mathrm{S}$ I and S II.

Texapon N70 (Cognis GmbH, Germany) is a sodium dodecyl ether sulphate (SDES) surfactant with a mass average molecular weight of $337 \mathrm{~g} / \mathrm{mol}$ as determined by ESI-MS, assuming equal ionizability of all species. Mainly chains with 12 and 14 carbon atoms and 0 to 3 ethylene oxide groups are present in the mixture. According to the supplier the main component is sodium 2-(2-dodecyloxyethoxy)ethyl sulfate. AOT [sodium-1,4-bis(2-ethylhexyl) sulfosuccinate, 98\%, see Fig. S3] was obtained from Sigma-Aldrich.

JR 400 (Dow Chemical, USA, see Fig. S4) is a cationically modified cellulose ether with a molecular weight of about $500000 \mathrm{~g} / \mathrm{mol}$ [26] and a degree of substitution of 0.27 , resulting in a charge density of $1000 \mathrm{~g}$ per mole of positive charges [27].

The samples with fully hydrogenated oil were diluted down to a concentration of $5 \mathrm{wt} \%$ oil-surfactant mixture in aqueous solution, where 0,2 (AOT) or $6.8 \mathrm{wt} \%$ (Texapon 
N70) of Tego Wipe DE (not the overall sample) have been replaced by one of the anionic surfactants. The solvent was either pure $\mathrm{D}_{2} \mathrm{O}$ (Eurisotop) or $\mathrm{D}_{2} \mathrm{O}$ in which $0.36 \mathrm{wt} \%$ of the $\mathrm{D}_{2} \mathrm{O}$ (not the overall sample) have been replaced by the cellulose-based polycation JR 400 (Dow Chemical). For simplicity these samples will be referred to as Tego Wipe NE (no additional surfactant), AOT NE (additional AOT) or Texapon NE (additional Texapon N70). Samples with additional JR 400 will be referred to as X/JR 400 , where $\mathrm{X}$ is the name of the additional surfactant.

The oil-surfactant mixture in the samples for contrast matched measurements consisted of $5 \mathrm{wt} \%$ of the oil-surfactant mixture P88 which has the same composition as Tego Wipe $\mathrm{DE}$, except that dilauryl citrate has been replaced by AOT since it was not commercially available. The exact composition is given in table S III and either hydrogenated DEHC (h-DEHC) or deuterated DEHC (d-DEHC) has been used. The solvent was a $1 / 9$ (wt/wt) mixture of $\mathrm{H}_{2} \mathrm{O}$ and $\mathrm{D}_{2} \mathrm{O}$ to match the contrast of the oil component with d-DEHC. For comparison the same sample but with hydrogenated DEHC has been measured. These samples will be referred to as d-DEHC NE and h-DEHC NE. The exact composition of all samples is given in Table S IV.

Fully deuterated DEHC (d-DEHC) was synthesized as follows (see Fig. S5): A solution of triphosgene in dichloromethane (DCM) was added dropwise to a solution of deuterated 2-ethylhexanol and pyridine in DCM. The solution was left to react overnight. Pyridine was removed by washing with an aqueous solution of hydrochloric acid twice. The organic phase was dried with $\mathrm{Na}_{2} \mathrm{SO}_{4}$ and the solvent was removed from the product by distillation. Details of the preparation and subsequent purification can be found elsewhere [28].

\section{B. Methods}

\section{Small-angle neutron scattering (SANS)}

SANS experiments were performed at the instruments D11 and D22 at the Institut Laue Langevin (ILL), Grenoble, France. To cover a large $Q$ range measurements were done at sample-detector distances of 1.2, 6, and $39 \mathrm{~m}$ (D11) and $1.4 \mathrm{~m}, 8$, and $17 \mathrm{~m}$ (D22). The wavelength was $6 \AA$, except for the $17 \mathrm{~m}$ configuration on D22, where a wavelength of $12 \AA$ was used. The transmission was measured with the attenuated direct beam and water was used to correct for the detector efficiency and to obtain absolute intensities. The software package BerSANS [29] was used for data reduction and radial averaging of the two dimensional scattering pattern.

\section{Neutron spin echo (NSE)}

The NSE experiments were performed on the instrument IN15 of the ILL, Grenoble (France) with wavelengths of (longest Fourier time in parentheses) 17 (250 ns), 10 (50 ns), $8 \AA$ ( $25 \mathrm{~ns})$ and $6 \AA$ (12.5 ns) to cover a $Q$ range from 0.12 to $1.91 / \mathrm{nm}$ and the instrument J-NSE of the FRMII, Garching (Germany) with wavelengths of 8 (33 ns) and $12.8 \AA$ (90 ns) to cover a $Q$ range from 0.36 to $1.51 / \mathrm{nm}$. Details of the experiments and the method are explained elsewhere [30-32].

\section{RESULTS AND DISCUSSION}

\section{A. Samples with core-shell structure}

The investigated system is a multicomponent system, where we concentrate on the already well-studied nanoemulsion range [9]. In order to obtain detailed structural and dynamical information we studied this system by means of SANS and NSE using both the hydrogenated and deuterated oil (DEHC).

As stabilizing ionic surfactant AOT was employed, as without such an ionic surfactant the stability of the nanoemulsions is significantly lower [28]. By using a $1 / 9$ mixture of $\mathrm{H}_{2} \mathrm{O}$ and $\mathrm{D}_{2} \mathrm{O}$ the solvent should match the contrast of the oil core of the droplets and highlight the surfactant membrane, which should also make membrane dynamics more visible. For comparison, the same sample but with hydrogenated DEHC (h-DEHC) has been measured as well, which should results in a homogeneous contrast profile of the droplets.

\section{SANS}

The SANS curves can be well described with two lognormal distributions of spheres using the same parameters of the distribution for both curves (see Fig. 1, Table S V and Ref. [9]), where for that concentration of $5 \mathrm{wt} \%$ the small microemulsion droplet distribution accounts for about $15 \mathrm{v} \%$, making our assumption, that we mainly observe dynamics of the larger nanoemulsion droplets well justified since its static scattering intensity exceeds that of the smaller microemulsion droplets by about a factor of at least 1.2 (see Fig. 2). According to the theory by Safran and Milner [33-35], which describes the membrane dynamics of the smaller microemulsion droplets in this $Q$ range the membrane dynamics can account for no more than about $20 \%$ of the signal, which is the case at the first minimum and would correspond to a factor 6 between the signal from the large droplets and the signal from the dynamics of the small droplets and we can safely neglect the membrane dynamics of the smaller droplets as in Eq. (11). For a more detailed discussion see Sec. II of the supporting information [25]. The minimum, which can be observed for the sample with d-DEHC at high $Q$ (Fig. 1, inset), shows that a core-shell structure is formed and the thickness of the shell is $1.6 \mathrm{~nm}$. To obtain good fit results, the contrast of the

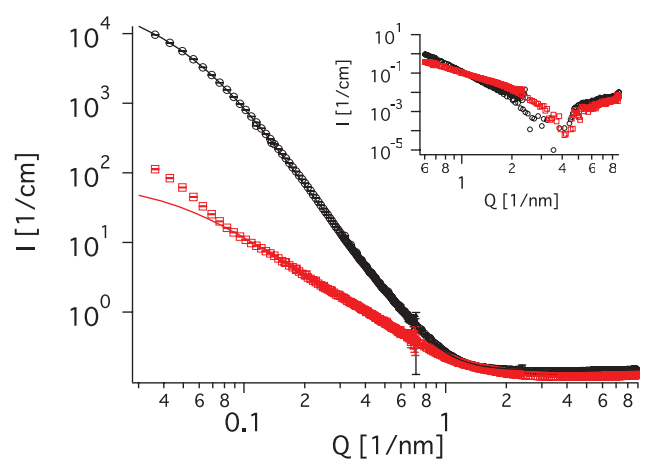

FIG. 1. (Color online) SANS curves of d-DEHC NE (red squares) and h-DEHC NE (black circles), fits: bimodal log-normal distribution of spheres, both curves are described with the same distribution; inset: high $Q$ with subtracted background, the shell thickness is determined from the minimum. 


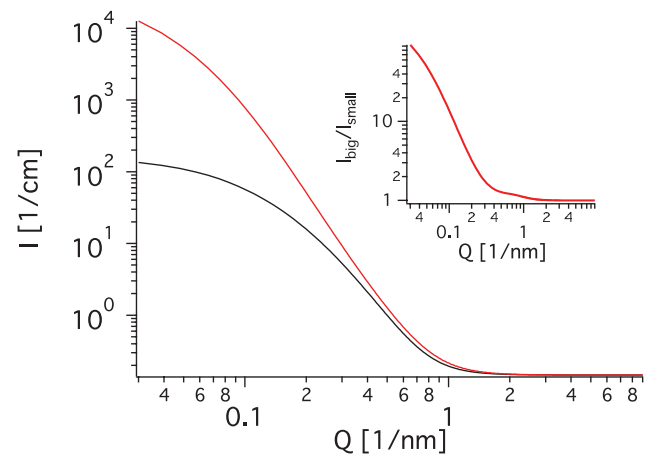

FIG. 2. (Color online) SANS intensities due to small particles (black) and big particles (red) obtained from the same parameters as in the fits for h-DEHC NE; inset: intensity ratio (big/small).

shell has to be somewhat reduced, which means that some intermixing of the cosurfactant (or oil) and the surfactant takes place in the shell. Interestingly, a similar minimum is observed for the sample with h-DEHC as well, which shows that even there some core-shell structure can be seen even though it is less pronounced due to the less favourable contrast. At low $Q$ some deviations become visible for the sample with deuterated oil, which may be attributed to some structure factor effects, since it is known that especially for polydisperse samples the effective structure may differ quite strongly for different contrast conditions and therefore may not be visible in bulk contrast, whereas it is visible in core-shell contrast [36]. These deviations should not play a role in the later analysis of NSE data, since such low- $Q$ values can not be reached by that method. The fitted standard deviations seem extremely high (see Table S V) but are necessary to obtain good fits as can be seen in Fig. S7, where the relative standard deviation has been fixed at $20 \%$. It is not clear, whether these high polydispersities are real or are just due to membrane fluctuations, which would make static scattering curves appear to be more polydisperse. Therefore, for analyzing NSE data, we employed both the obtained polydispersities and a relative standard deviation of $20 \%$ thereby making our analysis largely independent of the details of the prevailing polydispersity of the droplets.

\section{NSE}

The same two samples have been measured with NSE. The initial slope of $S(Q, t)$ has been fitted with a simple exponential to obtain an effective diffusion coefficient $D_{\text {eff }}$, which shows a $Q$ dependence (Fig. 3, data shown for d-DEHC NE). This may be due to an additional relaxation mode or just due to the polydispersity of the sample. That the latter is not the case here is shown by the line in Fig. 3, which represents the effective diffusion coefficient obtained from the initial slope, that would result from polydisperse droplets with the distribution obtained from SANS according to Eq. (8). In fact, an increase of $D_{\text {eff }}$ with $Q$ is observed, but at lower $Q$, where the ratio of the intensities between the two populations changes (see Fig. 2). To check this further, it was attempted to describe the obtained intermediate scattering functions with the full expression for diffusion of Eq. (8). Good fits are obtained indeed (see Fig. S9 and Fig. S10), using the distribution obtained from SANS, however the ratio between the two droplet populations

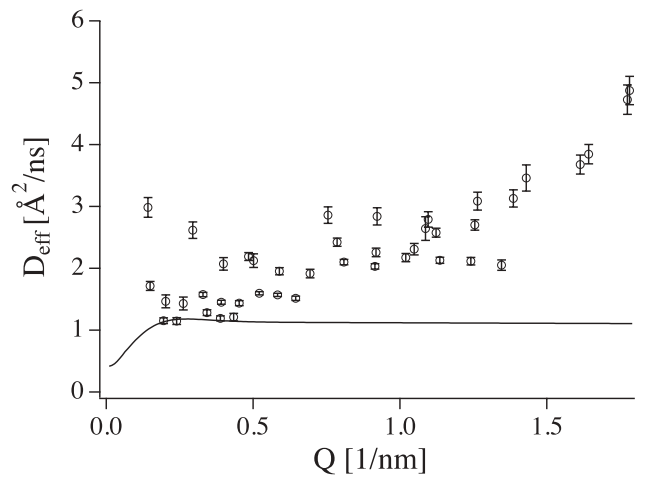

FIG. 3. Circles: Apparent diffusion coefficient $D_{\text {eff }}$ of d-DEHC NE. $D_{\text {eff }}$ increases linearly with $Q$, line: Diffusion coefficient, calculated from initial slope of Eq. (8) with parameters obtained from SANS, diffusion and polydispersity alone are not able to explain the data.

has to be left as a free parameter and attains significantly higher (and $Q$ dependent) values for the fraction of small droplets than in the SANS fits, which is $15 \mathrm{v} \%$ (see Fig. S8). This means that relaxation is faster than it should be, if only diffusion were present and this indicates that we do observe the fluctuations of the surfactant monolayer here. Applying Eq. (11) yields good fit results regardless of the polydispersity (see Figs. 4 and S14) but certainly the obtained values for $\Gamma_{k}$ and $a$ differ (see Fig. S11). It can also be seen that huge error bars result if both $a$ and $\Gamma_{k}$ are fit to individual curves. After an initial increase, $a$ takes a constant value between roughly 0.8 and $1.41 / \mathrm{nm}$ and increases again for even higher $Q$, probably because of shape fluctuations of the smaller droplets becoming visible. To obtain more stable values for $\Gamma_{k}$ the data was analyzed again with different fixed global values of $a$ (see Fig. 5). Regardless of $a, \Gamma_{k}$ takes more or less constant values between 0.8 and $1.41 / \mathrm{nm}$. This is probably the range in which our approximations (sufficiently large $Q$ to describe the membrane as more or less flat, sufficiently small $Q$ to neglect the dynamics of small droplets) hold. Since good results were obtained with constant $a$, its apparent $Q$ dependence is omitted and all $S(Q, t)$ data in the appropriate $Q$ range was fitted simultaneously to obtain $a$ and $\Gamma_{k}$ (and hence $\kappa$ ). Figures 6 and S16 show the data and fitted curves, confirming that this approach yields reliable values for $\Gamma_{k}$ and therefore allowing to deduce the mean bending modulus $\kappa$.

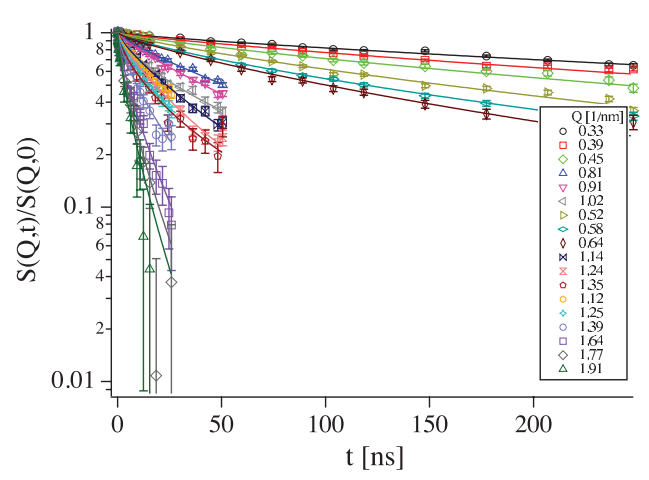

FIG. 4. (Color online) Intermediate scattering functions of dDEHC NE, fits taking into account fluctuations, high polydispersity. 


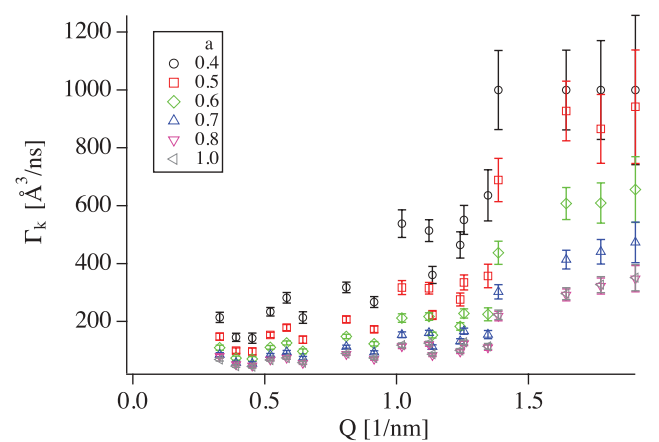

FIG. 5. (Color online) $\Gamma_{k}$ of d-DEHC NE for different (constant) values of $a$, as indicated in the graph, high polydispersity.

After it has been shown that information about the bending rigidity could be extracted from such a complex system with the aid of SANS and NSE in core-shell contrast, it has been attempted to apply the same scheme to the sample with hydrogenated oil in bulk contrast. In fact the qualitative behavior is very similar. Both $a$ and $\Gamma_{k}$ are more or less constant above $0.81 / \mathrm{nm}$ and good fit results are obtained with a single value of $a$ and $\Gamma_{k}$ (Figs. 7 and S17). The obtained fit parameters are summarized in Tables I and S VI. Using the high polydispersities obtained from SANS (able S V), the obtained $\Gamma_{k}$ for both bulk and core-shell contrast are in agreement within (the admittedly considerable) error, while there is a considerable discrepancy between the obtained $\kappa$ values if lower polydispersities are used. Namely, it appears that the sample with deuterated oil would have a very soft membrane, while the sample with hydrogenated oil would have a rather stiff membrane. This is unrealistic as the deuteration of the oil should basically have no effect on the elasticity of the membrane. Apparently only the use of the high polydispersities obtained from SANS leads to consistent results, even though there is a certain risk to treat some of the effects that are due to the dynamics of the membrane as polydispersity.

\section{B. Measurements with different ionic surfactants and encapsulation with the polycation JR 400}

After having established that membrane dynamics can be studied in such complex systems in both bulk and core-shell

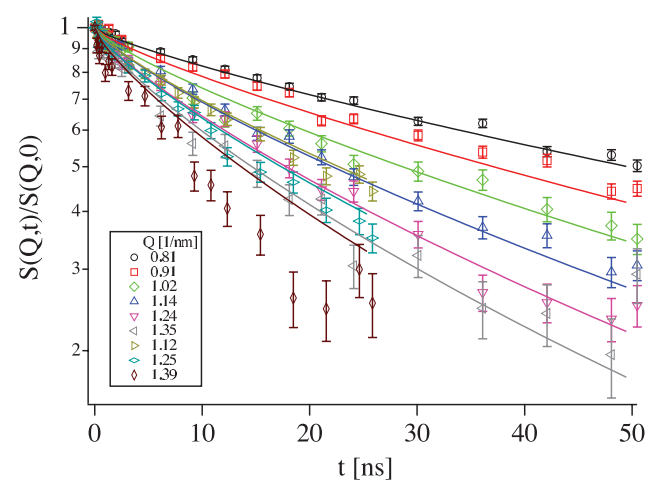

FIG. 6. (Color online) Intermediate scattering functions of dDEHC NE, simultaneous fits taking into account fluctuations, high polydispersity.

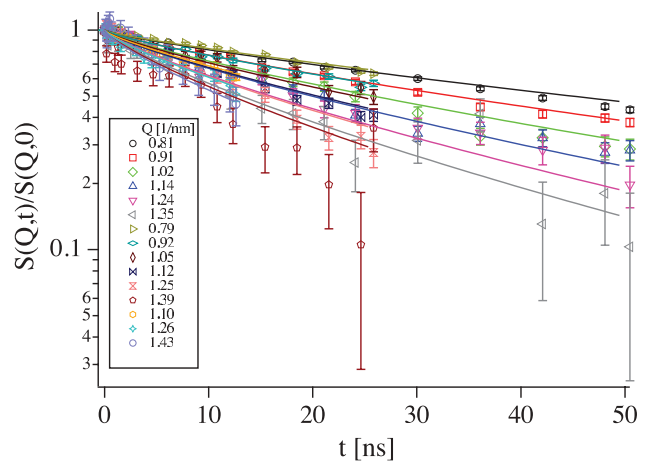

FIG. 7. (Color online) Intermediate scattering functions of hDEHC NE, simultaneous fits taking into account fluctuations, high polydispersity.

contrast, we went on and studied the influence of different anionic surfactants and addition of the cationic polyelectrolyte JR 400, which can be expected to attach to the surface of the oppositely charged nanoemulsion droplets, on the structure and dynamics of nanoemulsion systems. The exact conditions under which nanoemulsions are stable are still poorly understood, especially with respect to the effect of the individual molecular components (for instance in this case the choice of anionic surfactant). The idea behind the addition of JR 400 is its principal ability to encapsulate the nanoemulsion droplets, which might be a potentially promising approach to increase the stability of nanoemulsions, since it might stiffen the membrane and also prevent Ostwald ripening by preventing monomers from entering or leaving the droplets. In order to allow efficient encapsulation however the membrane must be charged in the first place and therefore a part of the original Tego Wipe DE formulation was replaced by an anionic surfactant (AOT or Texapon N70).

\section{SANS}

The SANS curves of the nanoemulsion systems with and without additional surfactant are shown in Fig. 8. The effect of additional JR 400 on nanoemulsions with AOT and Texapon N70 is shown in Fig. S22 and Fig. S23, respectively.

SANS data could be described with Eq. (6). The obtained fit parameters are summarized in Table II and show rather

TABLE I. Parameters obtained from NSE with high polydispersity, $Q$-independent Zilman-Granek relaxation rate $\Gamma_{k}$, bending modulus $\kappa$ and the amplitude of membrane dynamics $a$ [see Eq. (11)]. $\kappa$ is calculated with $\eta_{\text {eff }}=\eta 3$, its error $\Delta \kappa$ is $2 \Delta \Gamma_{k} / \Gamma_{k} \kappa$ and does not account for any systematic errors, $\Delta \kappa$ and $\Delta a$ are taken from the fits.

\begin{tabular}{lccc}
\hline \hline Sample & $\Gamma_{k}\left[\AA^{3} / \mathrm{ns}\right]$ & $\kappa[k T]$ & $a$ \\
\hline Tegowipe NE & $11 \pm 4$ & $8 \pm 5$ & $1.0 \pm 0.2$ \\
AOTJR 400 NE & $15 \pm 2$ & $4.2 \pm 0.9$ & $0.45 \pm 0.03$ \\
AOT NE & $13 \pm 1$ & $6 \pm 1$ & $0.63 \pm 0.03$ \\
Texapon/JR 400 NE & $17 \pm 1$ & $3.5 \pm 0.6$ & $1.00 \pm 0.04$ \\
Texapon NE & $17 \pm 1$ & $3.5 \pm 0.5$ & $1.00 \pm 0.04$ \\
d-DEHC NE & $10 \pm 2$ & $10 \pm 4$ & $0.82 \pm 0.08$ \\
h-DEHC NE & $8 \pm 2$ & $15 \pm 7$ & $1.0 \pm 0.1$ \\
\hline \hline
\end{tabular}




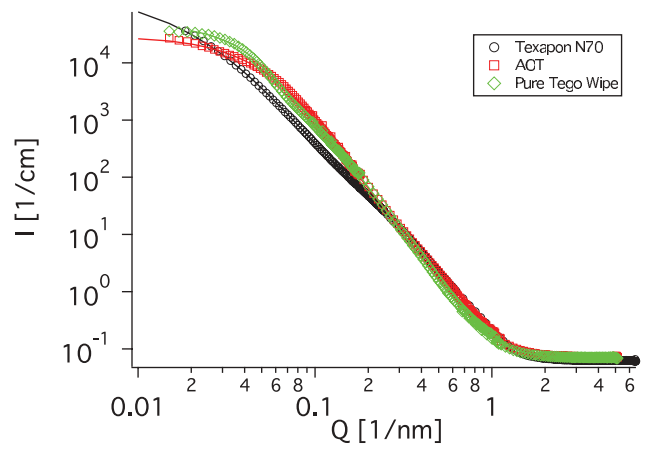

FIG. 8. (Color online) SANS curves of Texapon NE (black), AOT NE (red), and Tego Wipe NE (green).

insignificant changes compared to the systems without added JR400. Figure 9 shows the volume-weighted normalized size distributions of all five samples. While there are two welldistinguishable peaks for nanoemulsions without additional surfactant, AOT causes a shift of both peaks towards smaller radii. Also, the nanoemulsion droplets (second peak) become more polydisperse and their weight is increased. Addition of Texapon N70 causes the first peak to be shifted to smaller radii as well and in addition, the second peak becomes extremely smeared out. In fact, there is no more well-defined peak to be observed, which, of course, could also be due to our limited observation window in $Q$. For both AOT and Texapon N70, the addition of JR 400 has hardly any effect on the structure.

\section{NSE}

NSE data were evaluated with Eq. (11) in the same way as before (i.e., individual curves were fitted with different values of $a$ and $\Gamma_{k}$ ). Unless the result was obvious (i.e., the value of $a$ was the same for all curves when both $a$ and $\Gamma_{k}$ were free parameters of the fit), $\Gamma_{k}$ was fitted to the individual curves with different constant values of $a$ in order to see which range was suited for a fit with global parameters. In any case the suitable range turned out to be above $0.81 / \mathrm{nm}$. The curves obtained from the fits with global parameters are shown in Fig. 10 (AOT NE, shown here as a representative example), Fig. S18 (Tego Wipe NE), Fig. S19 (AOT/JR 400 NE), Fig. S20 (Texapon NE), and Fig. S21 (Texapon/JR 400 NE) and

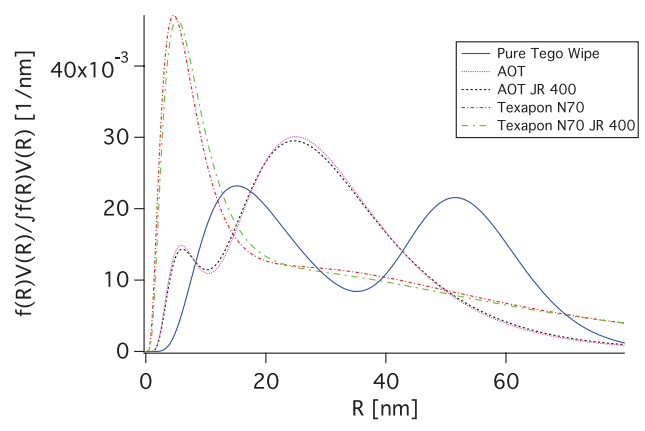

FIG. 9. (Color online) Normalized volume weight distributions of different samples, distributions obtained from SANS.

the fit parameters are summarized in Tables I and S VI. Good fits are always obtained, but to a lesser extent for the pure Tego Wipe Solution. This is probably due to the fact that the small distribution is somewhat larger here and the assumption of the membrane dynamics being only due to the larger distribution is less well fulfilled. The effect of the addition of the surfactants and JR 400 on the bending rigidity of the surfactant monolayer relative to the one of the pure Tego Wipe system is shown in Fig. 11, thereby avoiding the uncertainty concerning the absolute value of $\kappa$. It is interesting to note that the addition of ionic surfactant does not seem to stiffen the membrane as could be expected from a more strongly charged monolayer [37,38]. An explanation for this behavior would be that the addition of both ionic surfactants leads to an effectively thinner surfactant monolayer and as the mean-bending modulus $\kappa$ is proportional to the third power of the thickness $[39,40]$. This would have a pronounced effect on the observed modulus. The reduction is more pronounced for the Texapon N70 where also a higher degree of admixing was present.

Interestingly also, the addition of the oppositely charged JR 400 has basically no effect on the Texapon N70 doped system, while it leads some further reduction of $\kappa$ for the AOT system. The effects observed are within our nominal error bars, but it can be assumed that the error obtained from the fits is somewhat overestimated here, at least as far as the comparison of trends is concerned. A good part of the error bars is probably due to systematic errors, since our model is far from being perfect. However, since the size distributions

TABLE II. Parameters obtained from SANS with different surfactants and JR 400, macroscopic volume fraction $\phi_{m}$, fraction of material dissolved in bulk $f$ [so that $\phi=\phi_{m}(1-f)$ ], relative weight of first distribution $\phi_{\text {lrel }}$, mean of distribution x $M_{x}$ and its standard deviation $S_{D, x}$, scattering length density of material SLD (with $\Delta S_{\mathrm{LD}}=$ $S_{\mathrm{LD}}-S_{\mathrm{LD} \text {,bulk }}$, where $S_{\mathrm{LD} \text {,bulk }}$ is that of $\mathrm{D}_{2} \mathrm{O}$, modified by the dissolved material) and incoherent background (bkg).

\begin{tabular}{lccccc}
\hline \hline & Texapon/JR 400 NE & Texapon NE & AOT/JR 400 NE & AOT NE & Tego Wipe NE \\
\hline$\phi_{m}$ & 0.0597 & 0.0574 & 0.0599 & 0.0577 & 0.0578 \\
$f$ & 0.577 & 0.567 & 0.425 & 0.398 & 0.403 \\
$\phi_{1 \text { rel }}$ & 0.437 & 0.413 & 0.112 & 0.115 & 0.546 \\
$M_{1}[\mathrm{~nm}]$ & 2.58 & 2.25 & 4.21 & 4.12 & 9.79 \\
$S_{D_{1}}[\mathrm{~nm}]$ & 1.97 & 1.76 & 2.14 & 2.10 & 5.69 \\
$M_{2}[\mathrm{~nm}]$ & 17.5 & 17.8 & 18.5 & 18.9 & 49.80 \\
$S_{D_{2}}[\mathrm{~nm}]$ & 12.8 & 12.6 & 8.66 & 8.59 & 8.73 \\
$S_{\mathrm{LD}}\left[\mathrm{nm}^{-2}\right]$ & $3.39 \mathrm{E}-05$ & $3.38 \mathrm{E}-05$ & $3.14 \mathrm{E}-05$ & $3.13 \mathrm{E}-05$ & $3.07 \mathrm{E}-05$ \\
$\mathrm{bkg}\left[\mathrm{cm}^{-1}\right]$ & 0.0616 & 0.0628 & 0.0695 & 0.0732 & 0.0708 \\
\hline \hline
\end{tabular}




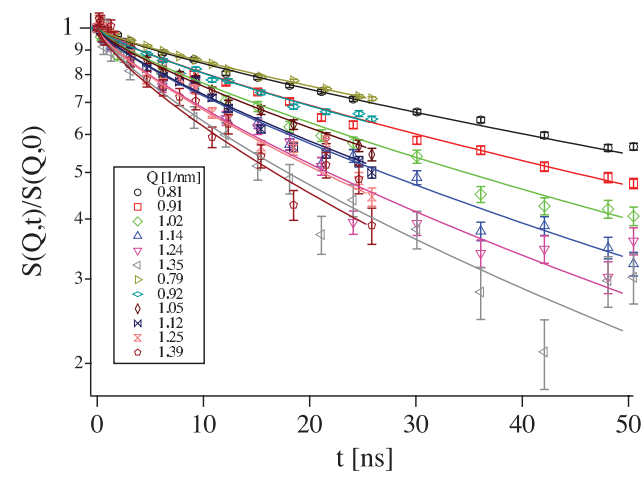

FIG. 10. (Color online) Intermediate scattering functions of AOT NE. Fits: fluctuation, shared $\Gamma_{k}$ and $a$, high polydispersity.

for samples with and without additional polyelectrolyte are very similar, the results are systematically wrong in the same manner. Therefore, at least the trends can be thought of as being significant. Interestingly, the membrane is softened, rather than stiffened by the addition of JR 400. From the data, it is not clear whether the polyelectrolyte is actually bound on the membrane, but it is likely due to the electrostatic conditions. In general, this effect can be attributed to an enhanced electrostatic screening, either by simply raising the ionic strength or compensating the charges directly by having JR 400 bound to the surface of the nanoemulsion droplets. An alternative explanation might be that by the presence of the polyelectrolyte some of the ionic surfactant now is bound to the polyelectrolyte in solution, thereby reducing the number of ionic surfactants in the amphiphilic monolayer, which renders it softer. In any case, apparently the polyelectrolyte-surfactant interaction is dominating over the effect that a thicker shell due to the bound polyelectrolyte has.

\section{Discussion}

The structure and dynamics of several nanoemulsion formulations obtained by the PIC method have been investigated using SANS and NSE. It could be shown that even in such complex and polydisperse systems, useful information can be gained concerning the dynamics of the membrane. It could be confirmed that a previously assumed [9] bimodal distribution

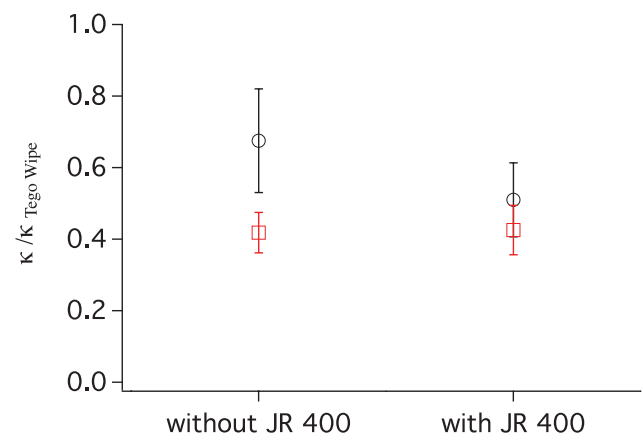

FIG. 11. (Color online) $\kappa$ relative to $\kappa$ of Tego Wipe NE for nanoemulsions with different added ionic surfactants, with or without JR 400, high polydispersity, black circles: AOT; red squares: Texapon N70. of spherical droplets is present in the solutions. In this work, parts of the basic nanoemulsion formulation were substituted by different ionic surfactants in order to encapsulate the charged droplets with an oppositely charged polyelectrolyte.

Using size distributions obtained from SANS, it could be shown that membrane dynamics can be observed with NSE, and can be described in terms of the bending elasticity using the model by Zilman and Granek [16]. SANS measurements with deuterated oil showed, that the droplets have a core-shell structure with a surfactant shell.

The addition of anionic surfactant (AOT or Texapon N70) leads to a shift of the bimodal distribution in different directions, which will be due to the different packing parameter of the single- and double-chain surfactant. From the NSE data we could deduce the bending modulus of the surfactant monolayer, which was reduced in a similar way by both anionic surfactants. This is presumably explained by an effectively thinner monolayer due to their incorporation into an otherwise rather stiff and thick monolayer formed by the polyglycerol4-laurate.

The addition of Texapon N70 results in more polydisperse droplets and less material is in the nanoemlusion as can be seen from the increase of $f$ (fraction of material dispersed in the bulk solution) and $\phi_{\text {1rel }}$ (fraction of material in the small droplets, see Table II). This is a bit surprising at first, especially when taking into account that there has been more Texapon N70 introduced in the system than AOT, which should result in a stronger charge of the droplets and thereby result in a higher-bending elasticity. But it might be argued that the single-chain ionic surfactant having a much smaller packing parameter [41] than AOT is not well suited to be incorporated in a relatively flat membrane such as the shell of the relatively large nanoemulsion droplets. Thus, most of the Texapon N70 might be in the small microemulsion droplets and the membrane of the nanoemulsion droplets actually is less charged and less stiff.

Interestingly adding oppositely charged polyelectrolyte has no visible effect on the structure of nanoemulsions, nor a larger effect on the stiffness of the surfactant monolayer. Only in nanoemulsions with additional AOT, a slight softening of the membrane can be observed with NSE.

These findings show that the combination of static and dynamic neutron scattering is very suitable to obtain a detailed structural and dynamical picture even for such relative complex colloidal systems as nanoemulsions. Apart from the bimodal size distribution information regarding the stiffness of the surfactant monolayer could be deduced and how it varies upon the modification of the system by added anionic surfactant or oppositely charged polyelectrolyte. This knowledge deepens the understanding of such systems and will allow in the future for a more rational approach in designing them.

\section{ACKNOWLEDGMENTS}

P.H. would like to thank the ILL for support through a Ph.D. scholarship, which allowed this work to be done. Financial support from the BMBF Project 05K10KT1 is gratefully acknowledged as well as allocation of beam time by the ILL and JCNS. 
[1] T. G. Mason, J. N. Wilking, K. Meleson, C. B. Chang, and S. M. Graves, J. Phys.: Condens. Matter 18, R635 (2006).

[2] T. Delmas, H. Piraux, A.-C. Couffin, I. Texier, F. Vinet, P. Poulin, M. E. Cates, and J. Bibette, Langmuir 27, 1683 (2011).

[3] K. Shinoda and H. Saito, J. Colloid Interface Sci. 26, 70 (1968).

[4] T. Förster, W. Von Rybinski, and A. Wadle, Adv. Colloid Interface Sci. 58, 119 (1995).

[5] P. Izquierdo, J. Feng, J. Esquena, T. F. Tadros, J. C. Dederen, M. J. Garcia, N. Azemar, and C. Solans, J. Colloid Interface Sci. 285, 388 (2005).

[6] C. Solans, P. Izquierdo, J. Nolla, N. Azemar, and M. GarciaCelma, Curr. Opin. Colloid Interface Sci. 10, 102 (2005).

[7] A. Maestro, I. Solè, C. González, C. Solans, and J. M. Gutiérrez, J. Colloid Interface Sci. 327, 433 (2008).

[8] I. Solè, C. M. Pey, A. Maestro, C. González, M. Porras, C. Solans, and J. M. Gutiérrez, J. Colloid Interface Sci. 344, 417 (2010).

[9] P. Heunemann, S. Prévost, I. Grillo, C. M. Marino, J. Meyer, and M. Gradzielski, Soft Matter 7, 5697 (2011).

[10] A. S. Kabalnov and E. D. Shchukin, Adv. Colloid Interface Sci. 38, 69 (1992).

[11] K. Landfester, N. Bechthold, F. Tiarks, and M. Antonietti, Macromolecules 32, 5222 (1999).

[12] T. Tadros, P. Izquierdo, J. Esquena, and C. Solans, Adv. Colloid Interface Sci. 108-109, 303 (2004).

[13] S. Clark, P. D. I. Fletcher, and X. Ye, Langmuir 6, 1301 (1990).

[14] J. Toro-Mendoza, A. Lozsan, M. Garcia-Sucre, A. J. Castellanos S., and G. Urbina-Villalba, Phys. Rev. E 81, 011405 (2010).

[15] M. Gradzielski, D. Langevin, and B. Farago, Phys. Rev. E 53, 3900 (1996).

[16] A. G. Zilman and R. Granek, Phys. Rev. Lett. 77, 4788 (1996).

[17] A. G. Zilman and R. Granek, Chem. Phys. 284, 195 (2002).

[18] W. Helfrich, Z. Naturforsch. C: J. Biosci. C 28, 693 (1973).

[19] M. Monkenbusch, O. Holderer, H. Frielinghaus, D. Byelov, J. Allgaier, and D. Richter, J. Phys.: Condens. Matter 17, S2903 (2005).

[20] S. Komura, T. Takeda, Y. Kawabata, S. K. Ghosh, H. Seto, and M. Nagao, Phys. Rev. E 63, 041402 (2001).

[21] M. C. Watson and F. L. H. Brown, Biophys. J. 98, L9 (2010).
[22] U. Seifert and S. A. Langer, Europhys. Lett. 23, 71 (1993).

[23] B. Farago, M. Monkenbusch, K. Goecking, D. Richter, and J. Huang, Phys. B 213, 214, 712 (1995).

[24] S. Wellert, M. Karg, O. Holderer, A. Richardt, and T. Hellweg, Phys. Chem. Chem. Phys. 13, 3092 (2011).

[25] See Supplemental Material at http://link.aps.org/supplemental/ 10.1103/PhysRevE.86.061407 for the chemical structure of compounds, the exact composition of samples, additional measurements and fit parameters and a more detailed discussion concerning the use of Eq. (11).

[26] S. Dhoot, E. Goddard, D. Murphy, and M. Tirrell, Colloids Surf. 66, 91 (1992).

[27] K. Thuresson, S. Nilsson, and B. Lindman, Langmuir 12, 530 (1996).

[28] P. Heunemann, Ph.D. thesis, Technische Universität, Berlin, 2011 [http://opus.kobv.de/tuberlin/volltexte/2012/3505/].

[29] U. Keiderling, Appl. Phys. A: Mater. Sci. Process. 74, S1455 (2002), Part 2 Suppl. S.

[30] P. Schleger, B. Alefeld, J. Barthelemy, G. Ehlers, B. Farago, P. Giraud, C. Hayes, A. Kollmar, C. Lartigue, F. Mezei, and D. Richter, Phys. B 241-243, 164 (1997).

[31] M. Monkenbusch, R. Schätzler, and D. Richter, Nucl. Instrum. Methods Phys. Res. Sect. A 399, 301 (1997).

[32] F. Mezei, in Lecture Notes in Physics, Vol. 128, edited by Ferenc Mezei (Springer, Berlin, 1980), pp. 1-26.

[33] S. A. Safran, J. Chem. Phys. 78, 2073 (1983).

[34] S. T. Milner and S. A. Safran, Phys. Rev. A 36, 4371 (1987).

[35] B. Farago and M. Gradzielski, J. Chem. Phys. 114, 10105 (2001).

[36] B. Weyerich, J. Brunner-Popela, and O. Glatter, J. Appl. Crystallogr. 32, 197 (1999).

[37] M. Winterhalter and W. Helfrich, J. Phys. Chem. 92, 6865 (1988).

[38] H. Lekkerkerker, Phys. A 159, 319 (1989).

[39] I. Szleifer, D. Kramer, A. Ben-Shaul, W. M. Gelbart, and S. A. Safran, J. Chem. Phys. 92, 6800 (1990).

[40] M. Gradzielski, D. Langevin, T. Sottmann, and R. Strey, J. Chem. Phys. 106, 8232 (1997).

[41] J. N. Israelachvili, D. J. Mitchell, and B. W. Ninham, J. Chem. Soc., Faraday Trans. II 72, 1525 (1976). 